\title{
Lupus eritematoso cutáneo juvenil subagudo con repercusión sistémica
}

Juvenile subacute cutaneous lupus erythematosus with systemic repercussions

\author{
Luz Vásquez ${ }^{1}$, Sneider Torres-Soto², Ana Cristina Ruiz ${ }^{3}$ \\ 1. Médica dermatóloga, Hospital Pablo Tobón Uribe, Medellín, Colombia \\ 2. Médico, Universidad Pontificia Bolivariana, Medellín, Colombia \\ 3. Médica dermatopatóloga, Hospital Pablo Tobón Uribe, Medellín, Colombia.
}

\section{RESUMEN}

Se reporta el caso de un paciente de sexo masculino de 12 años de edad que desarrolló un cuadro clínico de tres meses de evolución, consistente en la aparición de lesiones en placas en la piel: en la cara, el dorso nasal y las orejas, placas eritematosas anulares; en el tronco, la espalda y las extremidades superiores e inferiores, micropápulas eritematosas, algunas confluentes y no pruriginosas, y en la boca, aftas no dolorosas. Además, presentaba fotosensibilidad y síntomas constitucionales (fiebre y pérdida de peso).

En los exámenes paraclínicos, se encontró compromiso hematológico (leucopenia y linfopenia) y la prueba directa de Coombs fue positiva (sin signos de anemia) y hubo hipocomplementemia (C3 bajo). En el perfil inmunológico se informó la presencia de anticuerpos antinucleares positivos con patrón moteado y citoplásmico, y anticuerpos positivos contra antígenos nucleares extraíbles. La biopsia de piel demostró lupus eritematoso cutáneo subagudo.

Con estos hallazgos, se hizo diagnóstico de lupus eritematoso cutáneo subagudo que, posteriormente, evolucionó a lupus eritematoso sistémico, por lo cual requirió tratamiento con esteroides sistémicos.

PALABRAS CLAVE: lupus eritematoso cutáneo subagudo, lupus eritematoso sistémico, patrón anular, patrón psoriasiforme

\section{SUMMARY}

We report the case of a 12-year-old patient with a 3 month clinical history of plaques on skin of the face, nasal dorsum and ears, annular erythematous plaques in trunk, back, upper and lower extremities, erythematous micropapules, some confluent and not pruritic, and in mouth, painless canker sores. Also, had photosensitivity and constitutional symptoms (fever, weight loss)

Laboratory tests evidenced hematological compromise (leukopenia, lymphopenia), the direct Coombs test was positive (without anemia) and there was hypocomplementemia (low $\mathrm{C}_{3}$ ). The immunoserological profile found positive dappled and cytoplasmic antinuclear antibodies and positive antibodies against extractable nuclear antigens. The skin biopsy reported subacute cutaneous lupus erythematosus.

The patient met criteria for systemic lupus erythematosus and was treated

\author{
Correspondencia: \\ Sneider Alexander Torres \\ Email: \\ sneidertorreso7@gmail.com \\ Recibido: 11/08/2016 \\ Aceptado: 25/04/2017
}

Conflictos de interés:

No se reportan conflictos de interés.

Financiación:

Ninguna. 
with systemic steroids.

Key words: Lupus erythematosus, cutaneous, subacute; systemic lupus erythematosus; annular pattern; pattern psoriasiform
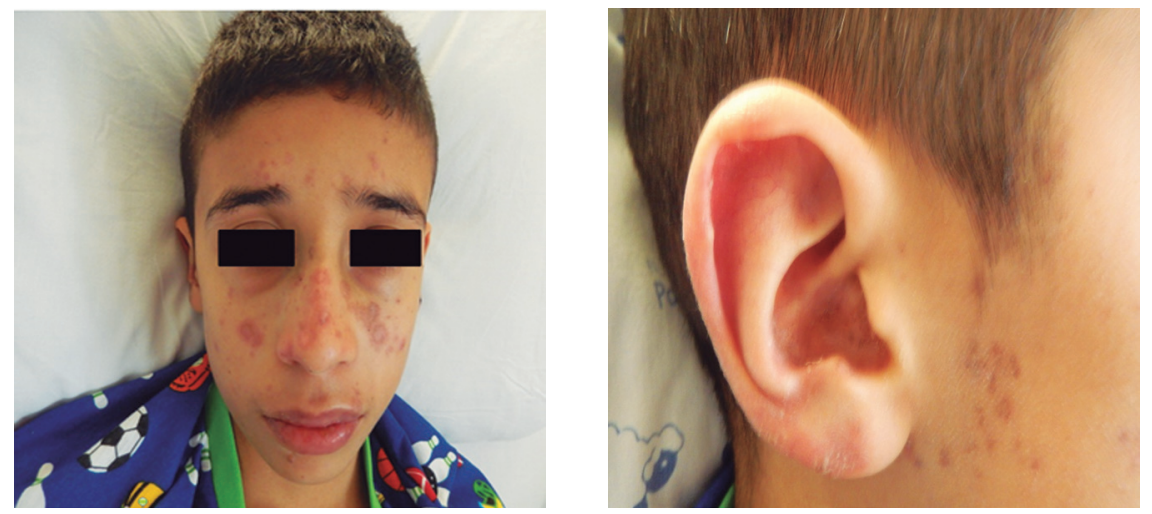

FIGURAS 1 Y 2. Placas anulares eritemato-violáceas, de bordes bien definidos, con descamación fina y ligera depresión central en la región malar, el dorso nasal y en la región preauricular.

\section{INTRODUCCIÓN}

El lupus eritematoso sistémico es una enfermedad autoinmunitaria con una amplia variedad de características clínicas, desde lesiones cutáneas limitadas hasta compromiso multiorgánico ${ }^{1}$. En la población infantil y juvenil, se estima una incidencia de 6 a 20 casos por 100.000 nacidos vivos ${ }^{2}$, con compromiso inferior al 8 $\%$ en menores de 14 años ${ }^{3}$. Se ha estimado que del 10 al $25 \%$ de los pacientes con lupus eritematoso sistémico desarrollan signos y síntomas durante esta época de la vida ${ }^{4}$.

El lupus cutáneo subagudo es un subgrupo que se caracteriza por presentar lesiones similares al lupus discoide, pero sin su componente evolutivo cicatricial y atrófico ${ }^{5}$.

\section{CASO CLÍNICO}

Se trata de un paciente de sexo masculino de 12 años de edad, sin antecedentes patológicos de importancia, que inicialmente presentó un cuadro clínico de tres meses de evolución, caracterizado por lesiones eritematosas en cara, manos y muslos, úlceras no dolorosas en la cavidad oral y fotosensibilidad. También, refirió sintomatología constitucional asociada, como fiebre vespertina subjetiva, pérdida de seis kilogramos de peso, malestar general, astenia y adinamia; también, coluria y oliguria. Este cuadro clínico no se asoció con fenómeno de Raynaud, alopecia, compromiso articular, serositis ni cefalea.

En el examen físico se observaron placas eritematovioláceas levemente infiltradas, con descamación superficial fina y centro más pálido, localizadas en el dorso nasal, las mejillas y la región preauricular (figuras 1 y 2). En los pulpejos y cara anterior del tercio proximal de los muslos, presentaba placas y pápulas eritemato-violáceas de bordes bien definidos (figuras 3 y 4). En la boca, se evidenciaron úlceras mucosas indoloras recubiertas con una membrana blanquecina, que comprometían el paladar y los carrillos.

En los exámenes de laboratorio al ingreso, se encontró: hemoglobina, 12,3 g/dl (valor de referencia: 12-16 g/ dl); hematocrito, 38,3 g/dl (valor de referencia: 30-45 g/dl) normales; leucopenia, 3.600 por mm3 (valor de referencia: 5.000-10.00o por $\mathrm{mm}_{3}$ ); linfopenia, 1.152 por mm3 (valor de referencia: 1.200-5.200 por mm3); plaquetas normales, 201.900 por mm3 (valor de referencia: 150.000-350.000 por $\mathrm{mm} 3$ ); pruebas de función hepática elevadas con patrón colestásico: TGO, 447 UI/L (valor de referencia: 5-55 UI/L), TGP, 133 UI/L (valor de referencia: 10-40 UI/L), FA, $96 \mathrm{UI} / \mathrm{L}$ (valor de referencia: 85-190 UI/L), GGT, $741 \mathrm{UI} / \mathrm{L}$ (valor de referencia: 8-37 UI/L); depuración de creatinina adecuada para la edad (134 ml/minuto/1,73 m2); relación proteinuria/creatinuria elevada ,1,52 $\mathrm{mg} / \mathrm{dl}$ (valor de referencia: < $1 \mathrm{mg} / \mathrm{dl}$ ); proteínas en orina de 24 horas levemente elevada, $160 \mathrm{mg} /$ día (valor de referencia: <150 mg/día); ANAS: patrón moteado, 1:320; patrón citoplásmico 1:160; anti-DNA negativos, ENAS anti-RNP, 83,3; anti-SM: 34,2, positivos (>20); anti-RO, 16,9; antiLA, 3,3, negativos (<20); anticuerpos anticardiolipina, IgG: 9,5, IgM: 11,6, negativos (<15); VDRL negativo; prueba directa de Coombs, positiva; virus hepatotropos negativos (HBV, HCV, EBV, CMV); HIV negativo, DENV negativo; electroforesis de proteínas sin alteraciones, $\mathrm{y}$ PPD negativo (2 mm).

Se practicaron biopsias de piel de la oreja y de las 


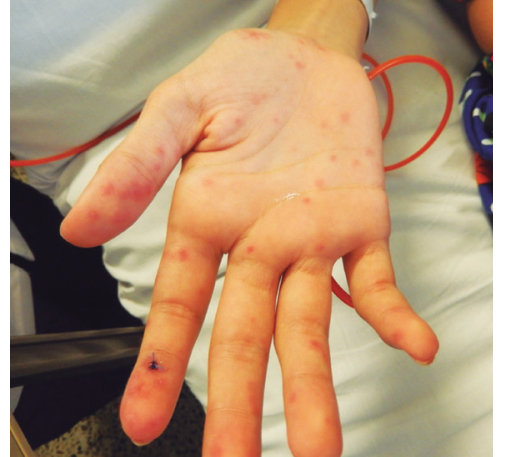

FIGURA 3. Pápulas y placas eritemato-violáceas con bordes bien definidos en los pulpejos. palmas. En la de la oreja, se observó aplanamiento epidérmico sin atrofia ni taponamiento folicular, vacuolas en la lámina basal e incremento de los queratinocitos necróticos, y en la dermis, leve infiltrado linfocitario perivascular superficial. Los hallazgos fueron similares en la biopsia de la palma, aunque más sutiles. Con estos resultados, se diagnosticó lupus cutáneo subagudo (figura 4).

Según los criterios clasificatorios de las Systemic Lupus International Collaborating Clinics (SLICC) para lupus eritematoso sistémico del 2012, el caso que se reporta satisface seis criterios: lupus cutáneo agudo (lupus cutáneo subagudo, fotosensibilidad), ANAS positivos, antígenos anti-Sm positivos, leucopenia, prueba directa de Coombs positiva en ausencia de anemia, e hipocomplementemia ( $\mathrm{C}_{3}$ bajo).

Con los hallazgos clínicos, paraclínicos e histopatológicos se hizo el diagnóstico de lupus eritematoso sistémico en el contexto de un paciente joven que presentó inicialmente lupus cutáneo subagudo, por lo que se inició tratamiento con cloroquina y prednisolona. Sin embargo, dada la elevación persistente de las enzimas hepáticas y el patrón colestásico, se practicó una resonancia magnética de abdomen en la que se observaron cambios inflamatorios hepáticos agudos, y en la biopsia de hígado se encontró hepatitis inespecífica; por lo tanto, se suspendió la cloroquina a los ocho días y se continuó con prednisolona como monoterapia.

\section{DISCUSIÓN}

El lupus eritematoso sistémico es una enfermedad autoinmunitaria, que puede presentar compromiso cutáneo hasta en $80 \%$ de los pacientes, uno de cada cuatro de ellos con lesiones cutáneas al momento del diagnóstico ${ }^{5}$.

El lupus cutáneo se caracteriza por un amplio espectro clínico, que puede tener manifestaciones específicas e inespecíficas de lupus cutáneo; según la clasificación

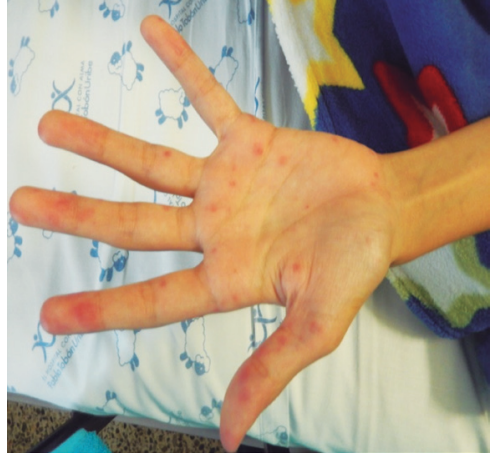

de Gilliam, el compromiso cutáneo específico se puede subdividir en tres tipos: lupus cutáneo agudo, subagudo y crónico ${ }^{1}$. Por otro lado, las manifestaciones inespecíficas, generalmente, se asocian con el lupus eritematoso sistémico, pero pueden asociarse con otras enfermedades, entre las que se destacan teleangiectasias periungulares, fenómeno de Raynaud, tromboflebitis, vasculitis leucocitoclástica, calcinosis cutis y eritema multiforme, entre otras ${ }^{6}$.

Los diferentes subtipos de lupus cutáneo se distinguen según sus manifestaciones cutáneas y su compromiso sistémico. El agudo se caracteriza por estar frecuentemente asociado el lupus eritematoso sistémico y presentarse en forma localizada o generalizada, mientras que el crónico presenta actividad sistémica en 5 a $10 \%$ de los casos y, según la presentación clínica, se subdivide en lupus discoide, profundo, sabañón o tumidus. El lupus cutáneo subagudo representa un espectro intermedio entre los dos subtipos anteriores ${ }^{7}$.

El lupus eritematoso cutáneo subagudo es un subtipo descrito por primera vez por Sontheimer, et al., en $1979^{8}$. Afecta un rango de edad estimado de 15 a 70 años ${ }^{9}$, con un importante predominio entre los 20 y los 40 años; muestra predilección por el sexo femenino, con una relación de mujer a hombre de 4,2:1 ${ }^{10}$ y es más común en personas caucásicas ${ }^{11}$.

Se estima que, aproximadamente, $50 \%$ de los pacientes con lupus eritematoso cutáneo subagudo cumplen cuatro o más criterios de la clasificación del American College of Rheumatology (ACR). Sin embargo, en algunos estudios se ha demostrado que solo 10 a $15 \%$ de los pacientes con lupus eritematoso cutáneo subagudo desarrollan manifestaciones orgánicas graves de evolución limitada ${ }^{6}$. Esta enfermedad produce fotosensibilidad hasta en $90 \%$ de los pacientes. Las lesiones cutáneas se inician como máculas o pápulas eritematosas que evolucionan hacia placas policíclicas o anulares (patrón anular) o lesiones pápulo-escamosas (patrón psoriasiforme) ${ }^{11}$, localizadas en áreas ex- 
puestas a la luz (cuello, hombros, extremidades superiores y tronco), y son menos comunes en las extremidades inferiores ${ }^{12}$. No se ha observado predominancia de ninguno de sus dos patrones: se ha informado que la mitad de los pacientes presenta el patrón psoriasiforme y, la otra mitad, el patrón anular. ${ }^{6}$

Con respecto a su perfil inmunológico, el lupus eritematoso cutáneo subagudo se caracteriza por la presencia de anticuerpos antinucleares positivos (ANA) en 60 a $80 \%$ de los pacientes, de anticuerpos anti-Ro en $70 \% \mathrm{y}$, en menor medida, de los anti-La en 30 a 50 $\%$ de los casos ${ }^{11}$. Cervera, et al., encontraron estos dos últimos anticuerpos positivos de forma conjunta en 70 $\%$ de los pacientes, con mayor frecuencia que cuando se compara con el lupus eritematoso sistémico ${ }^{3}$. Los anticuerpos anti-ADN, anti-Sm y anti-RNP son generalmente negativos en el $70 \%$ de los casos ${ }^{6}$.

El lupus cutáneo es poco común en la población pediátrica; en los niños, el riesgo de compromiso sistémico es mayor que en los adultos y son más frecuentes las presentaciones atípicas de la enfermedad ${ }^{13}$.

En el presente caso, se evidenció compromiso hematológico, cutáneo e inmunológico, lo cual permitió diagnosticar el lupus eritematoso sistémico.

Tiao, et al., en un análisis retrospectivo de 107 pacientes, observaron que los pacientes con lupus eritematoso cutáneo subagudo cumplían con los criterios del American College of Rheumatology, con los de las Systemic Lupus International Collaborating Clinics o con ambos, por predominio del compromiso mucocutáneo y por los parámetros de laboratorio; sin embargo, estos criterios no permiten diferenciar los pacientes con compromiso sistémico importante de quienes no lo presentan ${ }^{14}$.

Son pocos los casos reportados de lupus eritematoso cutáneo subagudo asociado a lupus eritematoso sistémico en la infancia. Frances, et al., reportaron una niña de dos meses de edad con diagnóstico de lupus cutáneo subagudo de patrón lineal (líneas de Blaschko), que progresó a lupus eritematoso sistémico ${ }^{15}$. Los reportes de casos de lupus eritematoso cutáneo sin evidencia de compromiso sistémico, son un poco más frecuentes. Buckley y Barnes reportaron un niño de nueve años de edad con lupus cutáneo subagudo, anticuerpos anti-Ro y anti-SSA positivos, y deficiencia de complemento $\left(\mathrm{C}_{2}\right)$, que clínicamente cursó con un exantema eritematoso, anular, con descamación e hipopigmentación periférica en las mejillas, la región palpebral y los pabellones auriculares. Rai, et al., reportaron un niño de 13 años de edad con lesiones eritematosas psoriasiformes en la cara y las extremidades, con una historia de fotosensibilidad de siete años de duración ${ }^{17}$. Otros casos similares se han reportado en la literatura científica ${ }^{18-20}$.

El tratamiento del lupus eritematoso cutáneo subagudo se enfoca en estrategias preventivas como la fotoprotección y en esquemas terapéuticos acordes con el compromiso sistémico subyacente. Los pacientes con lupus eritematoso sistémico requieren tratamiento sistémico convencional (corticosteroides, metotrexato, talidomida, retinoides, dapsona y azatioprina), nuevas terapias inmunomoduladoras o ambos. Por otro lado, los pacientes sin compromiso sistémico se benefician de los medicamentos tópicos (corticosteroides, inhibidores de calcineurina) y, según su reacción terapéutica, se determina si es necesario el tratamiento sistémico ${ }^{9,21}$. En el presente caso, el paciente presentó compromiso sistémico y requirió corticosteroides orales y cloroquina.

El pronóstico de la enfermedad está determinado por el compromiso sistémico. El tratamiento oportuno es vital para mejorar la evolución de la enfermedad y se estima que la tasa de supervivencia es de $90 \%$ a cinco años. En 62,5 \% de los pacientes, se presenta daño orgánico y tisular, de aparición más temprana en casos de enfermedad crónica activa ${ }^{22}$.

FigURA 4. Atrofia de la epidermis, degeneración vacuolar e infiltrado linfocitico perivascular. Hematoxilina y eosina, 4X y 40X
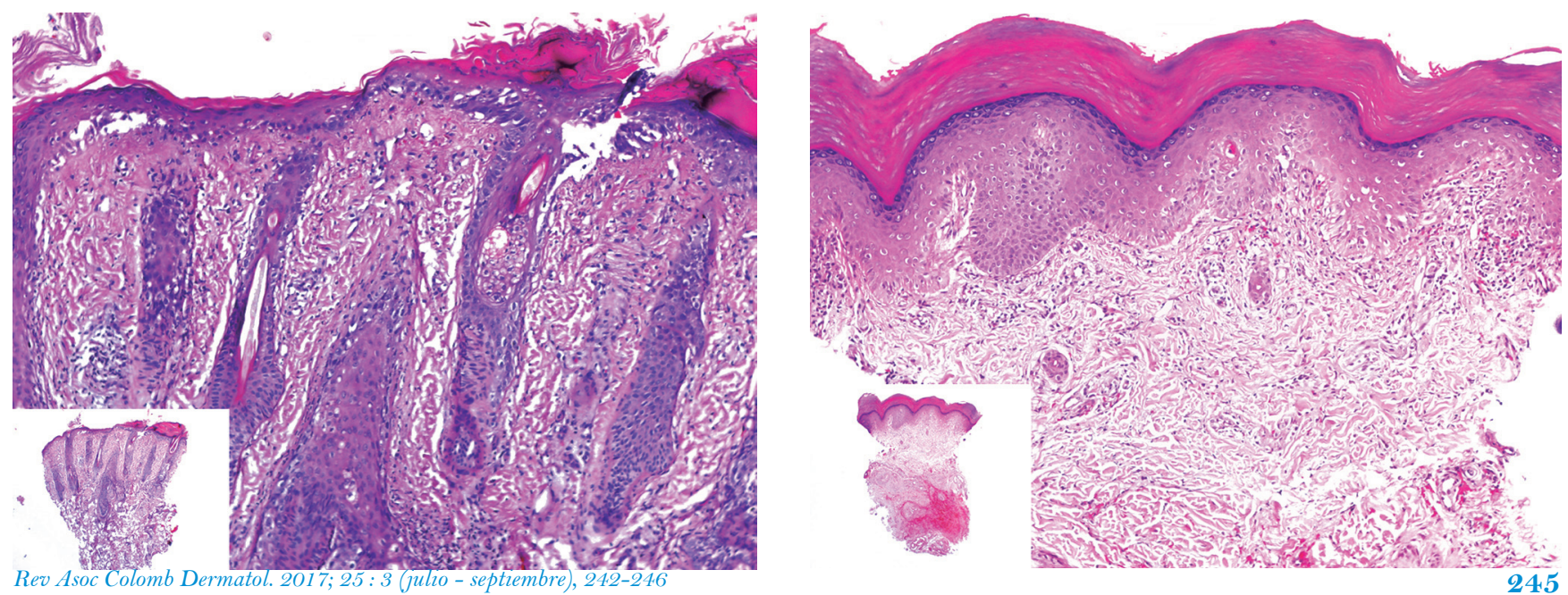


\section{CONCLUSIÓN}

El presente caso inicialmente hizo sospechar un lupus discoide; sin embargo, el paciente presentó compromiso sistémico y la biopsia de piel indicó un lupus cutáneo subagudo, poco usual para su edad y sexo, y de difícil diagnóstico clínico.

Como conclusión, el lupus eritematoso cutáneo subagudo es de baja prevalencia en la población pediátrica, y lo es más aun su progresión a lupus eritematoso sistémico, como en el presente caso. Se debe sospechar cuando se presentan lesiones cutáneas características y la enfermedad evoluciona con afectación sistémica.

\section{REFERENCIAS}

1. Hejazi EZ, Werth VP. Cutaneous lupus erythematosus: An update on pathogenesis, diagnosis and treatment. Am J Clin Dermatol. 2016;17:135-46.

2. Sztajnbok FR, Serra CRB, Rodrigues MCF, Mendoza E. Doenças reumáticas na adolescência. J Pediatr. 2001;77:234-44.

3. Cervera R, Khamashta MA, Font J, Sebastiani GD, Gil A, Lavilla P. Systemic lupus erythematosus: Clinical and immunologic pattern of disease expression in a cohort of 1000 patients. Medicine. 1993;72:113.

4. Ramírez LA, Uribe O, Osio O, Grisales H, Cardiel $\mathrm{MH}$, Wojdyla D, et al. Childhood systemic lupus erythematosus in Latin America. The GLADEL experience in 230 children. Lupus. 2008; 17:596-604.

5. Moura Filho JP, Peixoto RL, Martins LG, Melo SD, Carvalho LL, Pereira AK. Lupus erythematosus: Considerations about clinical, cutaneous and therapeutic aspects. An Bras Dermatol. 2014;89:11825.

6. Kuhn A, Landmann A. The classification and diagnosis of cutaneous lupus erythematosus. J Autoimmun. 2014;48-49:14-9.

7. Okon LG, Werth VP. Cutaneous lupus erythematosus: Diagnosis and treatment. Best Pract Res Clin Rheumatol. 2013;27:391-404.

8. Sontheimer RD, Thomas JR, Gilliam JN. Subacute cutaneous lupus erythematosus: A cutaneous marker for a distinct lupus erythematosus subset. Arch Dermatol. 1979;115:1409-15.

9. Brănişteanu DE, Lăbonţu A, Ciobanu D, Stoleriu G, Brănişteanu D, Oanţă A. Possible progression of subacute lupus erythematosus--case report. Rev Med Chir Soc Med Nat Iasi. 2014;118:381-6.

10. Li C, Wang B, Zhang J, Tan X. Clinical features, treatment and follow-ups of childhood systemic lupus erythematosus. Zhonghua Yi Xue Za Zhi. 2014;94:3259-61.

11. Grönhagen C, Nyberg F. Cutaneous lupus erythematosus: An update. Indian Dermatol Online J. 2014;5:7-13.

12. Sontheimer RD, Maddison PJ, Reichlin M, Jordon RE, Stastny P, Gilliam JN. Serologic and HLA association in subacute cutaneous lupus erythematosus: A clinical subset of lupus erythematosus. Ann Intern Med. 1982;97:664-71.

13. AlKharafi NN, Alsaeid K, AlSumait A, Al-Sabah $\mathrm{H}$, Al-Ajmi H, Rahim JA, et al. Cutaneous lupus erythematosus in children: Experience from a tertiary care pediatric dermatology clinic. Pediatr Dermatol. 2016;33:200-8.

14. Tiao J, Feng R, Carr K, Okawa J, Werth VP. Using the American College of Rheumatology (ACR) and Systemic Lupus International Collaborating Clinics (SLICC) criteria to determine the diagnosis of systemic lupus erythematosus (SLE) in patients with subacute cutaneous lupus erythematosus (SCLE). J Am Acad Dermatol. 2016;74:862-9.

15. Frances L, Betlloch I, Leiva-Salinas M, Marin I, Pascual JC. Subacute cutaneous lupus erythematosus starting as linear lupus erythematosus. Int J Dermatol. 2016;55:173-6.

16. Buckley D, Barnes L. Childhood subacute cutaneous lupus erythematosus associated with homozygous complement 2 deficiency. Pediatr Dermatol. 1995;12:327-330.

17. Rai VM, Balachandran C. Subacute cutaneous lupus erythematosus (SCLE) presenting in childhood. Dermatol Online J. 2005;11:27.

18. Mseddi M, Dammak A, Marrekchi S, Bouassida S, Zahaf A, Turki H. Subacute cutaneous lupus erythematosus in childhood: A case report. Arch Pediatr. 2007;14:164-6.

19. Amato L, Coronella G, Berti S, Moretti S, Fabbri P. Subacute cutaneous lupus erythematosus in childhood. Pediatr Dermatol. 2003;20:31-4.

20. Schoch JJ, Peters MS, Reed AM, Tollefson MM. Pediatric subacute cutaneous lupus erythematosus: Report of three cases. Int J Dermatol. 2015;54:e16974 .

21. Walling HW, Sontheimer RD. Cutaneous lupus erythematosus: Issues in diagnosis and treatment. Am J Clin Dermatol. 2009;10:365-81.

22. Sato JO, Corrente JE, Saad-Magalhães C. Chronic active disease pattern predicts early damage in juvenile systemic lupus erythematosus. Lupus. 2015;24:1421-8. 